\title{
ON THE DISTRIBUTION OF MIXED SUM OF INDEPENDENT RANDOM VARIABLES ONE OF THEM ASSOCIATED WITH SRIVASTAVA'S POLYNOMIALS AND $\bar{H}$-FUNCTION
}

\author{
JAGDEV SINGH AND DEVENDRA KUMAR
}

\begin{abstract}
In this paper, we obtain the distribution of mixed sum of two independent random variables with different probability density functions. One with probability density function defined in finite range and the other with probability density function defined in infinite range and associated with product of Srivastava's polynomials and $\overline{\mathrm{H}}$-function. We use the Laplace transform and its inverse to obtain our main result. The result obtained here is quite general in nature and is capable of yielding a large number of corresponding new and known results merely by specializing the parameters involved therein. To illustrate, some special cases of our main result are also given.
\end{abstract}

Mathematics Subject Classification 2010: 62E99, 60E05, 60E99, 33C60

Key Words and Phrases: $\bar{H}$-function, Srivastava's polynomials, Laplace transform, distribution function, probability density function

\section{INTRODUCTION}

In recent years, the distribution of sum of random variables has gained great importance in many areas of science and engineering. For example, sums of independent gamma random variables have application in problems of queuing theory such as determination of total waiting time, in civil engineering such as determination of the total excess water flow in a dam. They also appear in obtaining the inter arrival time of drought events which is the sum of the drought duration and the successive non drought duration. In last several years many authors notably Linhart [12], Jackson [11] and Grice and Bain [7] have studied the applications of distribution of sum of random variables. The distribution of the sum of two independent random variables has been obtained by many research workers, particularly when both the variates come from the same family of distribution. In this context the works of Albert [1] for uniform variates, Holm and Alouini [9], Moschopoulos [15] and Provost [17] for gamma variates, Van-Dorp and Kotz [23] for triangular variates (sharing the same support) and Loaiciga and Leipnik [13] for Gumbel variates are worth mentioning. 
Furthermore, Nason [16] has obtained the distribution of the sum of $t$ and Gaussian random variables and pointed out its application in Bayesian wavelet shrinkage. Very recently, Chaurasia and Singh [3] and Gupta [8] have studied the distribution of mixed sum of two independent random variables with different probability density functions. We know that the distribution of sum of several independent random variables when each random variable is of simply infinite or doubly infinite range can easily be obtained by means of characteristic function or moment generating function. But, when the random variables are distributed over finite range, these methods are not much useful and the power of integral transform method comes sharply into focus. In the present article, we obtain the distribution of sum of two independent random variables, $\mathrm{X}_{1}$ and $\mathrm{X}_{2}$, where $X_{1}$ possess finite uniform probability density function and $X_{2}$ follows infinite probability density function involving the product of Srivastava's polynomials and $\overline{\mathrm{H}}$ function, given by the equations (1) and (2) respectively.

Thus

$$
f_{1}\left(x_{1}\right)= \begin{cases}\frac{1}{a}, & 0 \leq x \leq a \\ 0, & \text { otherwise } a>0\end{cases}
$$

and

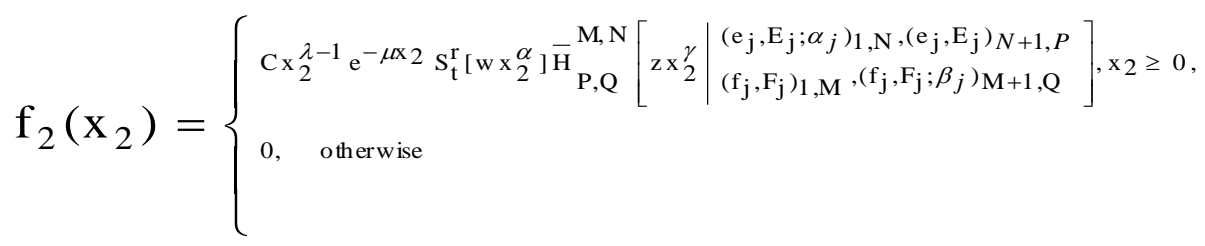

where

$$
\begin{aligned}
\mathrm{C}^{-1}=\mu^{-\lambda} & \sum_{\mathrm{k}=0}^{[\mathrm{t} / \mathrm{r}]} \frac{(-\mathrm{t})_{\mathrm{rk}}}{\mathrm{k} !} \mathrm{A}_{\mathrm{t}, \mathrm{k}} \mathrm{W}^{\mathrm{k}} \mu^{-\alpha \mathrm{k}} \\
& \times \overline{\mathrm{H}}_{\mathrm{P}+1, \mathrm{Q}}^{\mathrm{M}, \mathrm{N}+1}\left[\left.\mathrm{Z} \mu^{-\gamma}\right|_{\left(\mathrm{f}_{\mathrm{j}}, \mathrm{F}_{\mathrm{j}}\right)_{1, \mathrm{M}},\left(\mathrm{f}_{\mathrm{j}}, \mathrm{F}_{\mathrm{j}} ; \beta_{j}\right)_{\mathrm{M}+1, \mathrm{Q}}} ^{\left(\mathrm{e}_{\mathrm{j}}, \mathrm{E}_{\mathrm{j}} ; \alpha_{j}\right)_{1, \mathrm{~N}},(1-\lambda-\alpha \mathrm{k}, \gamma ; 1),\left(\mathrm{e}_{\mathrm{j}}, \mathrm{E}_{\mathrm{j}}\right)_{N+1, P}}\right]
\end{aligned}
$$

and the following conditions are satisfied:

$$
\begin{aligned}
& \gamma>0, \mu>0, \lambda+\alpha \mathrm{k}+\gamma \min _{1 \leq \mathrm{j} \leq \mathrm{M}}\left(\frac{\mathrm{f}_{\mathrm{j}}}{\mathrm{F}_{\mathrm{j}}}\right)>0, \\
& \mathrm{~A}=\sum_{\mathrm{j}=1}^{\mathrm{M}} \mathrm{F}_{\mathrm{j}}+\sum_{\mathrm{j}=1}^{\mathrm{N}} \mathrm{E}_{\mathrm{j}} \alpha_{j}-\sum_{\mathrm{j}=\mathrm{M}+1}^{\mathrm{Q}} \mathrm{F}_{\mathrm{j}} \beta_{j}-\sum_{\mathrm{j}=\mathrm{N}+1}^{\mathrm{P}} \mathrm{E}_{\mathrm{j}}>0,
\end{aligned}
$$


(iii) The parameters of $\overline{\mathrm{H}}$-function and Srivastava's polynomials are real and so restricted that $\mathrm{f}_{2}\left(\mathrm{x}_{2}\right)$ remains non-negative.

The $\overline{\mathrm{H}}$-function, a generalization of Fox H-function [6] introduced by Inayat Hussain [10] and studied by Buschman and Srivastava [2] and others, is defined and represented in the following manner:

$$
\begin{aligned}
\overline{\mathrm{H}}_{\mathrm{P}, \mathrm{Q}}^{\mathrm{M}, \mathrm{N}}[\mathrm{z}] & =\overline{\mathrm{H}}_{\mathrm{P}, \mathrm{Q}}^{\mathrm{M}, \mathrm{N}}\left[\mathrm{z} \mid \begin{array}{l}
\left(\mathrm{e}_{\mathrm{j}}, \mathrm{E}_{\mathrm{j}} ; \alpha_{j}\right)_{1, \mathrm{~N}},\left(\mathrm{e}_{\mathrm{j}}, \mathrm{E}_{\mathrm{j}}\right)_{N+1, P} \\
\left(\mathrm{f}_{\mathrm{j}}, \mathrm{F}_{\mathrm{j}}\right)_{1, \mathrm{M}},\left(\mathrm{f}_{\mathrm{j}}, \mathrm{F}_{\mathrm{j}} ; \beta_{j}\right)_{\mathrm{M}+1, \mathrm{Q}}
\end{array}\right] \\
& =\frac{1}{2 \pi \mathrm{i}} \int_{-\mathrm{i} \infty}^{+\mathrm{i} \infty} \bar{\phi}(\xi) \mathrm{z}^{\xi} \mathrm{d} \xi,
\end{aligned}
$$

where

$$
\bar{\phi}(\xi)=\frac{\prod_{\mathrm{j}=1}^{\mathrm{M}} \Gamma\left(\mathrm{f}_{\mathrm{j}}-\mathrm{F}_{\mathrm{j}} \xi\right) \prod_{\mathrm{j}=1}^{\mathrm{N}}\left\{\Gamma\left(1-\mathrm{e}_{\mathrm{j}}+\mathrm{E}_{\mathrm{j}} \xi\right)\right\}^{\alpha_{j}}}{\prod_{\mathrm{j}=\mathrm{M}+1}^{\mathrm{Q}}\left\{\Gamma\left(1-\mathrm{f}_{\mathrm{j}}+\mathrm{F}_{\mathrm{j}} \xi\right)\right\}^{\beta_{j}} \prod_{\mathrm{j}=\mathrm{N}+1}^{\mathrm{P}} \Gamma\left(\mathrm{e}_{\mathrm{j}}-\mathrm{E}_{\mathrm{j}} \xi\right)},
$$

and the contour $\mathrm{L}$ is the line from $\mathrm{C}-\mathrm{i} \infty$ to $\mathrm{C}+\mathrm{i} \infty$, suitably indented to keep poles of $\Gamma\left(\mathrm{f}_{\mathrm{j}}-\mathrm{F}_{\mathrm{j}} \xi\right), \mathrm{j}=1,2, \ldots, \mathrm{M}$ to the right of the path and the singularities of $\left\{\Gamma\left(1-\mathrm{e}_{\mathrm{j}}+\mathrm{E}_{\mathrm{j}} \xi\right)\right\}^{\alpha_{\mathrm{j}}}, \mathrm{j}=1,2, \ldots, \mathrm{N}$ to the left of the path. For convergence conditions and other details of the $\overline{\mathrm{H}}$-function see Inayat Hussain [10] and Buschman and Srivastava [2].

The Srivastava's polynomials [19] is defined as

$$
S_{t}^{r}[w]=\sum_{k=0}^{[t / r]} \frac{(-t)_{r k}}{k !} A_{t, k} w^{k}, \quad t=0,1,2, \ldots
$$

where $\mathrm{r}$ is an arbitrary positive integer, the coefficients $\mathrm{A}_{\mathrm{t}, \mathrm{k}}(\mathrm{t}, \mathrm{k} \geq 0)$ are arbitrary constants, real or complex.

\section{DISTRIBUTION OF THE MIXED SUM OF TWO INDEPENDENT RANDOM VARIABLES}

THEOREM 1. If $X_{1}$ and $X_{2}$ are two independent random variables having the probability density function defined by (1) and (2) respectively. Then the probability density function of 


$$
\mathrm{Y}=\mathrm{X}_{1}+\mathrm{X}_{2}
$$

is given by $\mathrm{g}(\mathrm{y})=\mathrm{g}_{1}(\mathrm{y}), 0 \leq \mathrm{y} \leq \mathrm{a}$

$$
=\mathrm{g}_{1}(\mathrm{y})-\mathrm{g}_{2}(\mathrm{y}), \mathrm{a}<\mathrm{y}<\infty
$$

where

$$
\begin{aligned}
& \mathrm{g}_{1}(\mathrm{y})=\frac{\mathrm{C}}{\mathrm{a}} \sum_{\mathrm{k}=0}^{[\mathrm{t} / \mathrm{r}]} \sum_{\mathrm{n}=0}^{\infty} \frac{(-\mathrm{t})_{\mathrm{rk}}}{\mathrm{k} !} \mathrm{A}_{\mathrm{t}, \mathrm{k}} \mathrm{w}^{\mathrm{k}} \mathrm{y}^{\lambda+\alpha \mathrm{k}} \frac{(-\mu \mathrm{y})^{\mathrm{n}}}{\mathrm{n} !} \\
& \times \overline{\mathrm{H}}_{\mathrm{P}+1, \mathrm{Q}+1}^{\mathrm{M}, \mathrm{N}+1}\left[\left.\mathrm{z} \mathrm{y}^{\gamma}\right|_{\left(\mathrm{f}_{\mathrm{j}}, \mathrm{F}_{\mathrm{j}}\right)_{1, \mathrm{M}},\left(\mathrm{f}_{\mathrm{j}}, \mathrm{F}_{\mathrm{j}} ; \beta_{j}\right)_{\mathrm{M}+1, \mathrm{Q}}(-\lambda-\alpha \mathrm{k}-\mathrm{n}, \gamma ; 1)} ^{\left(\mathrm{e}_{\mathrm{j}}, \mathrm{E}_{\mathrm{j}} ; \alpha_{j}\right)_{1, \mathrm{~N}},(1-\lambda-\alpha \mathrm{k}-\mathrm{n}, \gamma ; 1),\left(\mathrm{e}_{\mathrm{j}}, \mathrm{E}_{\mathrm{j}}\right)_{N+1, P}}\right], \mathrm{y} \geq 0
\end{aligned}
$$

and

$$
\begin{aligned}
\mathrm{g}_{2}(\mathrm{y}) & =\frac{\mathrm{C}}{\mathrm{a}} \sum_{\mathrm{k}=0}^{\mathrm{t} / \mathrm{r}]} \sum_{\mathrm{n}=0}^{\infty} \frac{(-\mathrm{t})_{\mathrm{rk}}}{\mathrm{k} !} \mathrm{A}_{\mathrm{t}, \mathrm{k}} \mathrm{w}^{\mathrm{k}}(\mathrm{y}-\mathrm{a})^{\lambda+\alpha \mathrm{k}} \frac{\{-\mu(\mathrm{y}-\mathrm{a})\}^{\mathrm{n}}}{\mathrm{n} !} \\
& \times \overline{\mathrm{H}}_{\mathrm{P}+1, \mathrm{Q}+1}^{\mathrm{M}, \mathrm{N}+1}\left[\left.\mathrm{z}(\mathrm{y}-\mathrm{a})^{\gamma}\right|_{\left(\mathrm{f}_{\mathrm{j}}, \mathrm{F}_{\mathrm{j}}\right)_{1, \mathrm{M}},\left(\mathrm{f}_{\mathrm{j}}, \mathrm{F}_{\mathrm{j}} ; \beta_{j}\right)_{\mathrm{M}+1, \mathrm{Q}}(-\lambda-\alpha \mathrm{k}-\mathrm{n}, \gamma ; 1)} ^{\left(\mathrm{e}_{\mathrm{j}}, \mathrm{E}_{\mathrm{j}} ; \alpha_{j}\right)_{1, \mathrm{~N}},(1-\lambda-\alpha \mathrm{k}-\mathrm{n}, 1),\left(\mathrm{e}_{\mathrm{j}}, \mathrm{E}_{\mathrm{j}}\right)_{N+1, P}}\right], \mathrm{y} \geq \mathrm{a}
\end{aligned}
$$

$\mathrm{C}$ is given by (3) and the following conditions are satisfied:

$$
\begin{aligned}
& \gamma>0, \mu>0, \lambda+\alpha \mathrm{k}+\gamma \min _{1 \leq \mathrm{j} \leq \mathrm{M}}\left(\frac{\mathrm{f}_{\mathrm{j}}}{\mathrm{F}_{\mathrm{j}}}\right)>0, \\
& \mathrm{~A}=\sum_{\mathrm{j}=1}^{\mathrm{M}} \mathrm{F}_{\mathrm{j}}+\sum_{\mathrm{j}=1}^{\mathrm{N}} \mathrm{E}_{\mathrm{j}} \alpha_{j}-\sum_{\mathrm{j}=\mathrm{M}+1}^{\mathrm{Q}} \mathrm{F}_{\mathrm{j}} \beta_{j}-\sum_{\mathrm{j}=\mathrm{N}+1}^{\mathrm{P}} \mathrm{E}_{\mathrm{j}}>0,
\end{aligned}
$$

(iii) The parameters of $\overline{\mathrm{H}}$-function and Srivastava's polynomials are real and so restricted that $\mathrm{g}_{1}(\mathrm{y})$ and $\mathrm{g}_{2}(\mathrm{y})$ remains non-negative.

PROOF. Let the Laplace transform of Y be denoted by $\phi_{\mathrm{y}}(\mathrm{s})$, then

$$
\phi_{\mathrm{y}}(\mathrm{s})=\mathrm{L}\left\{\mathrm{f}_{1}\left(\mathrm{x}_{1}\right) ; \mathrm{s}\right\} \mathrm{L}\left\{\mathrm{f}_{2}\left(\mathrm{x}_{2}\right) ; \mathrm{s}\right\}
$$

The Laplace transform of $\mathrm{f}_{1}\left(\mathrm{x}_{1}\right)$ is a simple integral so it can easily be evaluated and for the Laplace transform of $\mathrm{f}_{2}\left(\mathrm{x}_{2}\right)$, we express the $\overline{\mathrm{H}}$-function in terms of Mellin-Barnes type contour integral (5) and the Srivastava's polynomials in form of series (7). Further, we interchange the order of summation, $\mathrm{x}_{2}-$ and $\xi$-integrals and evaluate $\mathrm{x}_{2}$-integral as gamma integral to get 


$$
\begin{aligned}
& \phi_{\mathrm{y}}(\mathrm{s})=\frac{\mathrm{C}}{\mathrm{a}} \frac{\left(1-\mathrm{e}^{-\mathrm{as}}\right)}{\mathrm{s}} \sum_{\mathrm{k}=0}^{[\mathrm{t} / \mathrm{r}]} \frac{(-\mathrm{t})_{\mathrm{rk}}}{\mathrm{k} !} \mathrm{A}_{\mathrm{t}, \mathrm{k}} \mathrm{w}^{\mathrm{k}}(\mathrm{s}+\mu)^{-(\lambda+\alpha \mathrm{k})}
\end{aligned}
$$

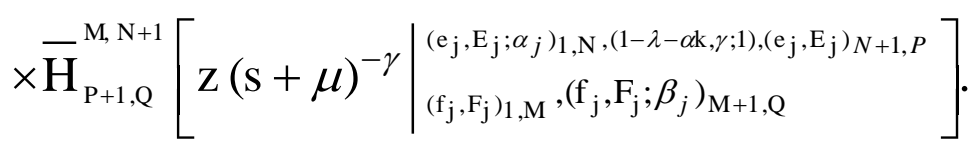

Now, we break the above expression in two parts, as follows

$$
\begin{aligned}
\phi_{\mathrm{y}}(\mathrm{s})= & \frac{\mathrm{C}}{\mathrm{a}} \sum_{\mathrm{k}=0}^{[\mathrm{t} / \mathrm{r}]} \frac{(-\mathrm{t})_{\mathrm{rk}}}{\mathrm{k} !} \mathrm{A}_{\mathrm{t}, \mathrm{k}} \mathrm{w}^{\mathrm{k}} \frac{(\mathrm{s}+\mu)^{-(\lambda+\alpha \mathrm{k})}}{\mathrm{s}} \\
& \times \overline{\mathrm{H}}_{\mathrm{P}+1, \mathrm{Q}}^{\mathrm{M}, \mathrm{N}+1}\left[\left.\mathrm{Z}(\mathrm{s}+\mu)^{-\gamma}\right|_{\left(\mathrm{f}_{\mathrm{j}}, \mathrm{F}_{\mathrm{j}}\right)_{1, \mathrm{M}},\left(\mathrm{f}_{\mathrm{j}}, \mathrm{F}_{\mathrm{j}} ; \beta_{j}\right)_{\mathrm{M}+1, \mathrm{Q}}} ^{\left.\left(\mathrm{e}_{\mathrm{j}} ; \alpha_{j}\right)_{1, \mathrm{~N}},(1-\lambda-\alpha \mathrm{k}, \gamma ; 1),\left(\mathrm{e}_{\mathrm{j}}, \mathrm{E}_{\mathrm{j}}\right)_{N+1, P}\right]}\right. \\
& -\frac{\mathrm{C}}{\mathrm{a}} \sum_{\mathrm{k}=0}^{[\mathrm{t} / \mathrm{r}]} \frac{(-\mathrm{t})_{\mathrm{rk}}}{\mathrm{k} !} \mathrm{A}_{\mathrm{t}, \mathrm{k}} \mathrm{w}^{\mathrm{k}} \frac{\mathrm{e}^{-\mathrm{as}}(\mathrm{s}+\mu)^{-(\lambda+\alpha \mathrm{k})}}{\mathrm{s}} \\
& \times \overline{\mathrm{H}}_{\mathrm{P}+1, \mathrm{Q}}^{\mathrm{M}, \mathrm{N}+1}\left[\left.\mathrm{Z}(\mathrm{S}+\mu)^{-\gamma}\right|_{\left.\left.\left(\mathrm{e}_{\mathrm{j}}, \mathrm{F}_{\mathrm{j}}\right)_{1, \mathrm{M}}, \mathrm{E}_{\mathrm{j}} ; \alpha, \mathrm{f}_{\mathrm{j}}, \mathrm{F}_{\mathrm{j}} ; \beta_{j}\right)_{\mathrm{M}+1, \mathrm{Q}},(1-\lambda-\alpha \mathrm{k}, \gamma ; 1),\left(\mathrm{e}_{\mathrm{j}}, \mathrm{E}_{\mathrm{j}}\right)_{N+1, P}\right] .}\right.
\end{aligned}
$$

To obtain the inverse Laplace transform of first term of equation (15), we express the $\overline{\mathrm{H}}$ function in contour integral, collect the terms involving ' $\mathrm{s}$ ' and take its inverse Laplace transform and use the known result (Erdélyi [4], p. 238, eq. 9). Further, writing the confluent hypergeometric function thus obtained in series form and interpreting the result by equation (5), we get the value of $g_{1}(y)$. The inverse Laplace transform of second term easily follows by the value of $\mathrm{g}_{1}(\mathrm{y})$ and shifting property for Laplace transform.

\section{SPECIAL CASES}

I. If we take $\alpha_{j}=1(j=1, \ldots N)$ and $\beta_{j}=1(j=M+1, \ldots, Q)$ in the main theorem, we arrive at the results recently obtained by Chaurasia and Singh [3].

II. If we set $t=0$ and $A_{0,0}=1$ in the main theorem, we get the results derived by Gupta [8].

III. Further, if we take $\alpha_{j}=1(j=1, \ldots N), \beta_{j}=1(j=M+1, \ldots, Q), t=0$ and $A_{0,0}=1$ in the main theorem, the pdf $\mathrm{f}_{2}\left(\mathrm{x}_{2}\right)$ reduces to the pdf defined by Mathai and Saxena [14] as follows 


$$
\mathbf{f}_{2}\left(\mathrm{X}_{2}\right)= \begin{cases}\mathrm{C}_{1} \mathrm{x}_{2}^{\lambda-1} \mathrm{e}^{-\mu \mathrm{x}_{2}} \mathrm{H}_{\mathrm{P}, \mathrm{Q}}^{\mathrm{M}, \mathrm{N}}\left[\mathrm{zx} \mathrm{zx}_{2}^{\gamma} \mid \begin{array}{l}
\left(\mathrm{e}_{\mathrm{j}}, \mathrm{E}_{\mathrm{j}}\right)_{1, \mathrm{P}} \\
\left(\mathrm{f}_{\mathrm{j}}, \mathrm{F}_{\mathrm{j}}\right)_{1, \mathrm{Q}}
\end{array}\right], \mathrm{x}_{2} \geq 0 \\
0, \quad \text { otherwise }\end{cases}
$$

where

$$
\mathrm{C}_{1}^{-1}=\mu^{-\lambda} \mathrm{H}_{\mathrm{P}+1, \mathrm{Q}}^{\mathrm{M}, \mathrm{N}+1}\left[\begin{array}{l|l}
\mathrm{Z} \mu^{-\gamma} & \begin{array}{l}
\left(\mathrm{e}_{\mathrm{j}}, \mathrm{E}_{\mathrm{j}}\right)_{1, \mathrm{P}},(1-\lambda, \gamma) \\
\left(\mathrm{f}_{\mathrm{j}}, \mathrm{F}_{\mathrm{j}}\right)_{1, \mathrm{Q}}
\end{array}
\end{array}\right]
$$

and the corresponding pdf of $\mathrm{Y}$ as obtained from the equation (9) is given by

$$
\begin{aligned}
\mathrm{h}(\mathrm{y}) & =\mathrm{h}_{1}(\mathrm{y}), 0 \leq \mathrm{y} \leq \mathrm{a} \\
& =\mathrm{h}_{1}(\mathrm{y})-\mathrm{h}_{2}(\mathrm{y}), \mathrm{a}<\mathrm{y}<\infty
\end{aligned}
$$

where

$$
\mathrm{h}_{1}(\mathrm{y})=\frac{\mathrm{C}_{1}}{\mathrm{a}} \mathrm{y}^{\lambda} \sum_{\mathrm{n}=0}^{\infty} \frac{(-\mu \mathrm{y})^{\mathrm{n}}}{\mathrm{n} !} \mathrm{H}_{\mathrm{P}+1, \mathrm{Q}+1}^{\mathrm{M}, \mathrm{N}+1}\left[\left.\mathrm{z} \mathrm{y}^{\gamma}\right|_{\left(\mathrm{f}_{\mathrm{j}}, \mathrm{F}_{\mathrm{j}}\right)_{1, \mathrm{Q}},(-\lambda-\mathrm{n}, \gamma)} ^{\left(\mathrm{e}_{\mathrm{j}}, \mathrm{E}_{\mathrm{j}}\right)_{1, \mathrm{P}},(1-\lambda-\mathrm{n}, \gamma)}\right], \mathrm{y} \geq 0
$$

and

$$
\begin{aligned}
\mathrm{h}_{2}(\mathrm{y})= & \frac{\mathrm{C}_{1}(\mathrm{y}-\mathrm{a})^{\lambda}}{\mathrm{a}} \sum_{\mathrm{n}=0}^{\infty} \frac{\{-\mu(\mathrm{y}-\mathrm{a})\}^{\mathrm{n}}}{\mathrm{n} !} \\
& \times \mathrm{H}_{\mathrm{P}+1, \mathrm{Q}+1}^{\mathrm{M}, \mathrm{N}+1}\left[\left.\mathrm{z}(\mathrm{y}-\mathrm{a})^{\gamma}\right|_{\left(\mathrm{f}_{\mathrm{j}}, \mathrm{F}_{\mathrm{j}}\right)_{1, \mathrm{Q}},(-\lambda-\mathrm{n}, \gamma)} ^{\left(\mathrm{e}_{\mathrm{j}}, \mathrm{E}_{\mathrm{j}}\right)_{1, P},(1-\lambda-\mathrm{n}, \gamma)}\right], \mathrm{y} \geq \mathrm{a}
\end{aligned}
$$

where $\mathrm{C}_{1}$ is given by (17) and the same conditions are satisfied as given in Theorem 1.

IV. By applying Theorem 1 to the case of Hermite polynomials (Srivastava and Singh [20] and Sezgö [22]) and by setting

$$
\mathrm{S}_{\mathrm{t}}^{2}(\mathrm{w}) \rightarrow \mathrm{w}^{\mathrm{t} / 2} \mathrm{H}_{\mathrm{t}}\left[\frac{1}{2 \sqrt{\mathrm{w}}}\right]
$$

in which case $r=2, A_{t, k}=(-1)^{k}$, the pdf $f_{2}\left(x_{2}\right)$ assumes the following form

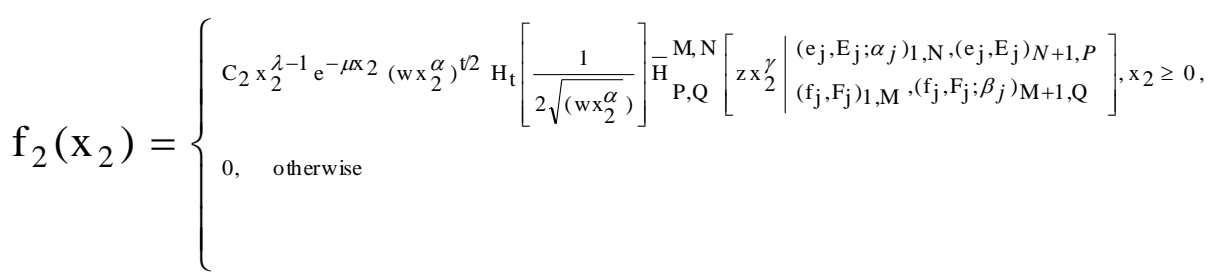


where

$$
\begin{aligned}
\mathrm{C}_{2}^{-1}= & \mu^{-\lambda} \sum_{\mathrm{k}=0}^{[\mathrm{t} / 2]} \frac{(-\mathrm{t})_{2 \mathrm{k}}}{\mathrm{k} !}(-1)^{\mathrm{k}} \mathrm{w}^{\mathrm{k}} \mu^{-\alpha \mathrm{k}} \\
& \times \overline{\mathrm{H}}_{\mathrm{P}+1, \mathrm{Q}}^{\mathrm{M}, \mathrm{N}+1}\left[\begin{array}{l|l}
\mathrm{Z} \mu^{-\gamma} & \begin{array}{l}
\left(\mathrm{e}_{\mathrm{j}}, \mathrm{E}_{\mathrm{j}} ; \alpha_{j}\right)_{1, \mathrm{~N}},(1-\lambda-\alpha \mathrm{k}, \gamma ; 1),\left(\mathrm{e}_{\mathrm{j}}, \mathrm{E}_{\mathrm{j}}\right)_{N+1, P} \\
\left(\mathrm{f}_{\mathrm{j}}, \mathrm{F}_{\mathrm{j}}\right)_{1, \mathrm{M}},\left(\mathrm{f}_{\mathrm{j}}, \mathrm{F}_{\mathrm{j}} ; \beta_{j}\right)_{\mathrm{M}+1, \mathrm{Q}}
\end{array}
\end{array}\right]
\end{aligned}
$$

and the corresponding pdf of $\mathrm{Y}$ as obtained from the equation (9) is given by

$$
\begin{aligned}
\mathrm{u}(\mathrm{y}) & =\mathrm{u}_{1}(\mathrm{y}), 0 \leq \mathrm{y} \leq \mathrm{a} \\
& =\mathrm{u}_{1}(\mathrm{y})-\mathrm{u}_{2}(\mathrm{y}), \mathrm{a}<\mathrm{y}<\infty
\end{aligned}
$$

where

$$
\begin{aligned}
\mathrm{u}_{1}(\mathrm{y})= & \frac{\mathrm{C}_{2}}{\mathrm{a}} \sum_{\mathrm{k}=0}^{[\mathrm{t} / 2]} \sum_{\mathrm{n}=0}^{\infty} \frac{(-\mathrm{t})_{2 \mathrm{k}}}{\mathrm{k} !}(-1)^{\mathrm{k}} \mathrm{w}^{\mathrm{k}} \mathrm{y}^{\lambda+\alpha \mathrm{k}} \frac{(-\mu \mathrm{y})^{\mathrm{n}}}{\mathrm{n} !} \\
& \times \overline{\mathrm{H}}_{\mathrm{P}+1, \mathrm{Q}+1}^{\mathrm{M}, \mathrm{N}+1}\left[\left.\mathrm{z} \mathrm{y}\right|_{\left(\mathrm{f}_{\mathrm{j}}, \mathrm{F}_{\mathrm{j}}\right)_{1, \mathrm{M}},\left(\mathrm{f}_{\mathrm{j}}, \mathrm{F}_{\mathrm{j}} ; \beta_{j}\right)_{\mathrm{M}+1, \mathrm{Q}}(-\lambda-\alpha \mathrm{k}-\mathrm{n}, \gamma ; 1)} ^{\left(\mathrm{e}_{\mathrm{j}}, \mathrm{E}_{\mathrm{j}} ; \alpha_{j}\right)_{1, \mathrm{~N}},(1-\lambda-\alpha \mathrm{k}-\mathrm{n}, \gamma ; 1),\left(\mathrm{e}_{\mathrm{j}}, \mathrm{E}_{\mathrm{j}}\right)_{N+1, P}}\right], \mathrm{y} \geq 0
\end{aligned}
$$

and

$$
\begin{aligned}
& \mathrm{u}_{2}(\mathrm{y})=\frac{\mathrm{C}_{2}}{\mathrm{a}} \sum_{\mathrm{k}=0}^{[\mathrm{t} / 2]} \sum_{\mathrm{n}=0}^{\infty} \frac{(-\mathrm{t})_{2 \mathrm{k}}}{\mathrm{k} !}(-1)^{\mathrm{k}} \mathrm{w}^{\mathrm{k}}(\mathrm{y}-\mathrm{a})^{\lambda+\alpha \mathrm{k}} \frac{\{-\mu(\mathrm{y}-\mathrm{a})\}^{\mathrm{n}}}{\mathrm{n} !}
\end{aligned}
$$

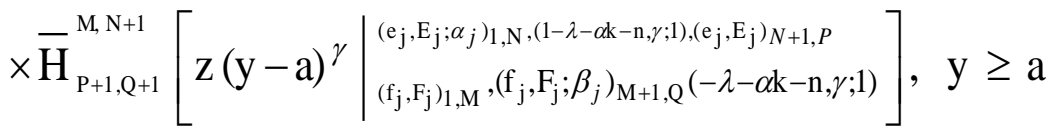

where $\mathrm{C}_{2}$ is given by (22) and the conditions are satisfied as obtainable from Theorem 1. Next, if we take $\alpha_{j}=1(j=1, \ldots N)$ and $\beta_{j}=1(j=M+1, \ldots, Q)$, we get a known result obtained earlier by Chaurasia and Singh [3].

V. By applying Theorem 1 to the case of Laguerre polynomials (Srivastava and Singh [20] and Sezgö [22]) and by setting

$$
\mathrm{S}_{\mathrm{t}}^{1}(\mathrm{w}) \rightarrow \mathrm{L}_{\mathrm{t}}^{(\sigma)}(\mathrm{w})
$$

in which case $\mathrm{r}=1, \mathrm{~A}_{\mathrm{t}, \mathrm{k}}=\left(\begin{array}{c}\mathrm{t}+\sigma \\ \mathrm{t}\end{array}\right) \frac{1}{(\sigma+1)_{\mathrm{k}^{\prime}}}$, the pdf $\mathrm{f}_{2}\left(\mathrm{x}_{2}\right)$ reduces to the following form 


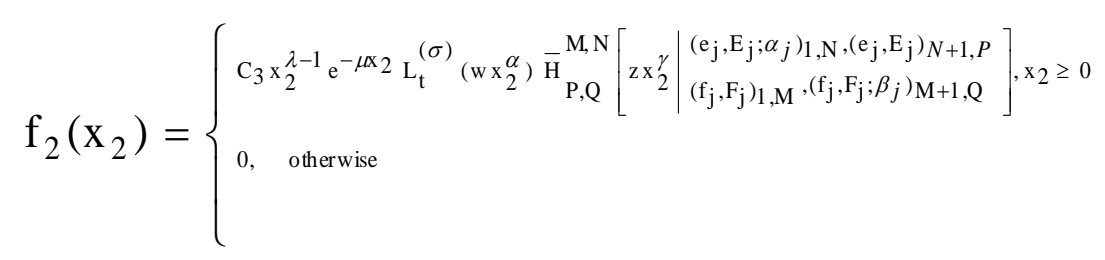

where

$$
\begin{aligned}
& \mathrm{C}_{3}^{-1}=\mu^{-\lambda} \sum_{\mathrm{k}=0}^{[\mathrm{t}]} \frac{(-\mathrm{t})_{2 \mathrm{k}}}{\mathrm{k} !}\left(\begin{array}{c}
\mathrm{t}+\sigma \\
\mathrm{t}
\end{array}\right) \frac{1}{(\sigma+1)_{\mathrm{k}^{\prime}}} \mathrm{w}^{\mathrm{k}} \mu^{-\alpha \mathrm{k}} \\
& \times \overline{\mathrm{H}}_{\mathrm{P}+1, \mathrm{Q}}^{\mathrm{M}, \mathrm{N}+1}\left[\begin{array}{l|l}
\mathrm{z} \mu^{-\gamma} & \begin{array}{l}
\left(\mathrm{e}_{\mathrm{j}}, \mathrm{E}_{\mathrm{j}} ; \alpha_{j}\right)_{1, \mathrm{~N}},(1-\lambda-\alpha \mathrm{k}, \gamma ; 1),\left(\mathrm{e}_{\mathrm{j}}, \mathrm{E}_{\mathrm{j}}\right)_{N+1, P} \\
\left(\mathrm{f}_{\mathrm{j}}, \mathrm{F}_{\mathrm{j}}\right)_{1, \mathrm{M}},\left(\mathrm{f}_{\mathrm{j}}, \mathrm{F}_{\mathrm{j}} ; \beta_{j}\right)_{\mathrm{M}+1, \mathrm{Q}}
\end{array}
\end{array}\right]
\end{aligned}
$$

and the corresponding pdf of $\mathrm{Y}$ as obtained from the equation (9) is given by

$$
\begin{aligned}
\mathrm{v}(\mathrm{y}) & =\mathrm{v}_{1}(\mathrm{y}), 0 \leq \mathrm{y} \leq \mathrm{a} \\
& =\mathrm{v}_{1}(\mathrm{y})-\mathrm{v}_{2}(\mathrm{y}), \mathrm{a}<\mathrm{y}<\infty
\end{aligned}
$$

where

$$
\begin{aligned}
& \mathrm{v}_{1}(\mathrm{y})=\frac{\mathrm{C}_{3}}{\mathrm{a}} \sum_{\mathrm{k}=0}^{[\mathrm{t}]} \sum_{\mathrm{n}=0}^{\infty} \frac{(-\mathrm{t})_{\mathrm{k}}}{\mathrm{k} !}\left(\begin{array}{c}
\mathrm{t}+\sigma \\
\mathrm{t}
\end{array}\right) \frac{1}{(\sigma+1)_{\mathrm{k}^{\prime}}} \mathrm{w}^{\mathrm{k}} \mathrm{y}^{\lambda+\alpha \mathrm{k}} \frac{(-\mu \mathrm{y})^{\mathrm{n}}}{\mathrm{n} !}
\end{aligned}
$$

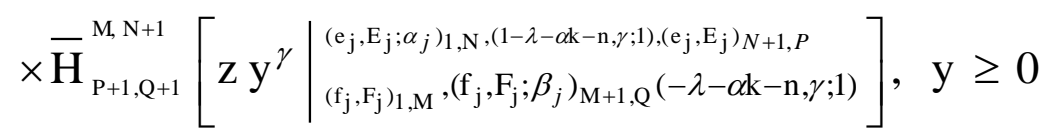

and

$$
\begin{aligned}
& \mathrm{v}_{2}(\mathrm{y})=\frac{\mathrm{C}_{3}}{\mathrm{a}} \sum_{\mathrm{k}=0}^{[\mathrm{t}]} \sum_{\mathrm{n}=0}^{\infty} \frac{(-\mathrm{t})_{\mathrm{k}}}{\mathrm{k} !}\left(\begin{array}{c}
\mathrm{t}+\sigma \\
\mathrm{t}
\end{array}\right) \frac{1}{(\sigma+1)_{\mathrm{k}^{\prime}}} \mathrm{w}^{\mathrm{k}}(\mathrm{y}-\mathrm{a})^{\lambda+\alpha \mathrm{k}} \frac{\{-\mu(\mathrm{y}-\mathrm{a})\}^{\mathrm{n}}}{\mathrm{n} !} \\
& \times \overline{\mathrm{H}}_{\mathrm{P}+1, \mathrm{Q}+1}^{\mathrm{M}, \mathrm{N}+1}\left[\left.\mathrm{z}(\mathrm{y}-\mathrm{a})^{\gamma}\right|_{\left(\mathrm{f}_{\mathrm{j}}, \mathrm{F}_{\mathrm{j}}\right)_{1, \mathrm{M}},\left(\mathrm{f}_{\mathrm{j}}, \mathrm{F}_{\mathrm{j}} ; \beta_{j}\right)_{\mathrm{M}+1, \mathrm{Q}}(-\lambda-\alpha \mathrm{k}-\mathrm{n}, \gamma ; 1)} ^{\left(\mathrm{e}_{\mathrm{j}} ; \mathrm{E}_{j}\right)_{1, \mathrm{~N}},(1-\lambda-\alpha \mathrm{k}-\mathrm{n}, \gamma ; 1),\left(\mathrm{e}_{\mathrm{j}}, \mathrm{E}_{\mathrm{j}}\right)_{N+1, P}}\right], \mathrm{y} \geq \mathrm{a}
\end{aligned}
$$

where $\mathrm{C}_{3}$ is given by (27) and the conditions are satisfied surrounding Theorem 1.

Further, if we set $\alpha_{j}=1(j=1, \ldots N)$ and $\beta_{j}=1(j=M+1, \ldots, Q)$, we get a known result derived by Chaurasia and Singh [3]. 
The importance of our result lies in its manifold generality. In view of the generality of the $\overline{\mathrm{H}}$-function and the Srivastava's polynomials, on specializing the various parameters in the $\overline{\mathrm{H}}$-function and the Srivastava's polynomials, we obtain, from our results, several pdfs such as the gamma pdf, beta pdf, Rayleigh pdf, Weibull pdf, Nakagami-m pdf, Chi-Squared pdf, half-Gaussian pdf, one-sided exponential pdf, halfCauchy pdf, lognormal pdf, Rician pdf, $\mathrm{K}_{v}$ pdf etc. and their distribution functions. Thus, the results presented in this paper would at once yield a very large number of pdfs occurring in the problems of science and engineering.

\section{ACKNOWLEDGMENTS}

The authors are grateful to the referee for his invaluable suggestions and comments for the improvement of the paper.

\section{REFERENCES}

[1] J. Albert, Sums of uniformly distributed variables, College Mathematics Journal, 33 (2002), 201-206.

[2] R.G. Buschman and H.M. Srivastava, The $\bar{H}$-Function Associated with a Certaion Class of Feynman Integrals, Journal of Physics A: Mathematical and General, 23 (1990), 4707-4710.

[3] V.B.L. Chaurasia and J. Singh, The distribution of the sum of mixed independent random variables pertaining to special functions, International Journal of Engineering Science and Technology, 2(7) (2010), 2601-2606.

[4] A. Erdélyi et al., Tables of Integral Transforms, Vol. I, McGraw-Hill, New York, (1954).

[5] A. Erdélyi et al., Higher Transcendental Functions, Vol. I. McGraw-Hill, New York, (1953).

[6] C. Fox, The $\mathrm{G}$ and $\mathrm{H}$ functions as symmetrical Fourier kernels, Trans. Amer. Math. Soc., 98 (1961), 395-429.

[7] J.V. Grice and L.J. Bain, Inferences concerning the mean of the gamma distribution, Journal of the American Statistical Association, 75 (1980), 929-933.

[8] M.K. Gupta, The distribution of mixed sum of independent random variables one of them associated with $\overline{\mathrm{H}}$-function, Ganita Sandesh, 22(2) (2008), 139-146.

[9] H. Holm, M.A. Alouini, Sum and difference of two squared correlated Nakagami variates in connection with the McKay distribution, IEEE Transactions on communications, 52(8) (2004), 1367-1376.

[10] A.A. Inayat-Hussain, New Properties of Hypergeometric Series Derivable from Feynman Integrals. II: A Generalization of the H-function, , Journal of Physics A: Mathematical and General, 20 (1987), 41194128.

[11] O.A.Y. Jackson, Fitting a gamma or log-normal distribution to fiber-diameter measurements on wool tops, Applied Statistics, 18(1969), 70-75.

[12] H. Linhart, Approximate confidence limits for the coefficient of variation of gamma distributions, Biometrics, 21 (1965), 733-738.

[13] H.A. Loaiciga and R.B. Leipnik, Analysis of extreme hydrologic events with Gumbel distributions: marginal and additive cases, Stochastic Environmental Research and Risk Assessment, 13 (1999), 251259.

[14] A.M. Mathai and R.K. Saxena, On the linear combination of stochastic variables, Metrika, 20(3) (1973), 160-169.

[15] P.G. Moschopoulos, The distribution of the sum of independent gamma random variables, Annals of the Institute of Statistical Mathematics, 37 (1985), 541-544.

[16] G.P. Nason, On the sum of $t$ and Gaussian random variables, Statistics and Probability Letters, 76(12) (2006), 1280-1286.

[17] S.B. Provost, On sums of independent gamma random variables, Statistics, 20 (1989), 589-591.

[18] M.D. Springer, The Algebra of Random Variables, John Wiley and Sons, New York, (1979).

[19] H.M. Srivastava, A contour integral involving Fox's H-function, Indian Journal of Mathematics, 14 (1972), 1-6. 
[20] H.M. Srivastava and N.P. Singh, The integration of certain products of the multivariable H-function with a general class of polynomials, Rend. Circ. Mat. Palermo, (Ser. 2) 32 (1983), 157-187.

[21] H.M. Srivastava and J.P. Signhal, On a class of generalized hypergeometric distributions, Jnanabha Sect. A 2 (1972), 1-9.

[22] C. Sezgö, Orthogonal polynomials, Amer. Math. Soc. Colloq. Publ. 23 Fourth edition, Amer. Math. Soc. Providence, Rhode Island, (1975).

[23] J.R. Van Dorp and S. Kotz, Generalizations of two sided power distributions and their convolution, Communications in Statistics, Theory and Methods, 32(9) (2003), 1703-1723.

\section{Jagdev Singh}

Department of Mathematics, Jagan Nath University,

Village-Rampura, Tehsil-Chaksu,

Jaipur-303901, Rajasthan, India

\section{Devendra Kumar}

Department of Mathematics, JaganNath Gupta Institute of Engineering and Technology, Jaipur-302022, Rajasthan, India

Received July 2012 